\title{
OCORRÊNCIA DE Oryzaephilus surinamensis LINNAEUS, 1758 (COLEOPTERA:CUCUJIDAE) E Necrobia rufipes DE GEER, 1775 (COLEOPTERA:CLERIDAE) INFESTANDO RAÇÕES DE ANIMAIS DOMÉSTICOS
}

\begin{abstract}
Rodrigo Gredilha(1), Priscilla Ribeiro Saavedra( ${ }^{(2)}$, Luciana Guerim ${ }^{(2)}$, Aurino Florêncio de Lima ${ }^{(1)} \&$ Nicolau Maués Serra-Freire ${ }^{(3)}$
\end{abstract}

\section{Abstract}

Occurrence of Oryzaephilus surinamensis Linnaeus, 1758 (Coleoptera:Cucujidae) e Necrobia rufipes De Geer, 1775 (Coleoptera:Cleridae) infesting pet food - The occurrence of insects associated with pet foods was verified in simultaneous collections of peletes accomplished after the opening of the sacks wrapped in the industry and distributed for commercialization, in the period of may to november of 2003. There were selected for analysis six different types of the total available marks for sale in the city of Rio de Janeiro. The incidence of the beetles Oryzaephilus surinamensis and Necrobia rufipes was testified.

Key words: Coleoptera, Necrobia rufipes, Oryzaephilus surinamensis, pet food

(1) Laboratório de Entomologia, Departamento de Entomologia e Fitopatologia, Instituto de Biologia, Universidade Federal Rural do Rio de Janeiro, km 47, CEP: 23.890-000 Seropédica, Rio de Janeiro, Brasil. E-mail: gredilha@ioc.fiocruz.br; (2) Universidade Estácio de Sá, Estrada Boca do Mato 850, CEP: 22783-320 Vargem pequena, Rio de Janeiro, Brasil; (3) Laboratório de Ixodides, Departamento de Entomologia, Instituto Oswaldo Cruz/FIOCRUZ, Av. Brasil 4365, CEP: 21045-900 Manguinhos, Rio de Janeiro, RJ, Brasil.

Recebido em: 04/11/2004.

Aceito em: 23/03/2005. 


\section{Resumo}

Verificou-se a ocorrência de insetos associados a rações industriais para cães e gatos. Coletas simultâneas de péletes foram realizadas após a abertura dos sacos embalados na indústria e distribuídos para comercialização, no período de maio à novembro de 2003. Foram selecionadas para análise, seis diferentes tipos do total das marcas disponíveis para a venda no comércio da cidade do Rio de Janeiro. Registrou-se a incidência dos besouros Necrobia rufipes e Oryzaephilus surinamensis.

Palavras-chave: Coleoptera, Necrobia rufipes, Oryzaephilus surinamensis, ração animal

\section{Introdução}

Veer et al. (1996) e Fields (1992) mencionaram que as diversas espécies de insetos associados a produtos armazenados são cosmopolitas adaptadas a climas extremamente variados apresentando altos índices de dispersão populacional. As pragas de produtos armazenados são citadas por Nakano et al. (2002) e Baggio et al. (1987) por apresentarem pouca exigência em relação à luz e temperatura, sendo favorecidas pelo ambiente do depósito que geralmente é escuro. O aumento da intensidade luminosa juntamente com o arejamento do local prejudica o desenvolvimento e reprodução destas pragas (Curtis \& Clark, 1974; Odeyemi, 1997; Buchelos \& Athanassiou, 1999).

Os adultos e as larvas de $O$. surinamensis infestam cereais, farinhas, frutos secos, chocolate, macarrão e até carnes defumadas (Nakano et al., 2002). Trata-se de uma praga secundária quando em grãos já infestados ou defeituosos (Howe, 1956). Necrobia rufipes está presente nos alimentos vegetais em conserva (Sengupta et al.,1984) ; amêndoa de côco (Nwana,1993 ; Veer et al., 1996) ; castanha de caju (Pratissoli, 1997) e com maior freqüência nos produtos de origem animal tal como peixes e carnes secas (Ashman, 1963; Osuji, 1977; Odeyemi, 1997). É também predador de outros insetos podendo promover canibalismo quando há superpopulação (Lambkin \& Khantoon, 1990; Veer et al., 1996). Há relatos deste inseto associado a carcaça de suínos e cadáveres humanos (Car- 
valho et al., 2000 ; Shalaby et al., 2000). Este coleóptero é considerado por Nwana (1993) e Simmons \& Ellington (1925) como uma praga primária de importância agro-industrial, com alto índice de dispersão populacional.

A suspeita de insetos infestando rações industriais ocorreu a partir das denúncias dos proprietários de cães e gatos em diferentes localidades da cidade do Rio de Janeiro que adquirem tais produtos em lojas especializadas. Apesar da notificação feita às empresas responsáveis pela distribuição e industrialização dos produtos, novos casos continuaram a surgir. A constatação da presença de insetos em lotes de diferentes marcas mobilizou os autores a recolherem amostras com o objetivo de identificar os insetos e verificar a provável ocorrência da infestação.

\section{Material e Métodos}

A partir da queixa dos consumidores estabeleceu-se como método para investigação o indutivo incompleto, usando como amostragem os locais de comercialização com valores modais de reclamações. Definiuse que o estudo seria só para casos no município do Rio de Janeiro restringindo-se $o$ recolhimento das amostras em seis tipos diferentes de marcas a venda no comércio e com embalagem industrial no período de maio à novembro de 2003, sendo recolhida em cada marca uma amostra mensal totalizando assim, 42 amostras

A amostragem consistiu em coleta de $1,5 \mathrm{~kg}$ dos péletes das rações logo após a abertura dos sacos no local de comercialização. Devido a dispersão dos insetos dentro dos pacotes, houve a necessidade de coletar $0,5 \mathrm{~kg}$ em cada um terço das embalagens originais que contém $15 \mathrm{Kg}$. Os insetos (adultos e larvas) encontrados nas rações foram manualmente removidos e preservados em recipientes contendo solução de etanol $70 \%$. Por questões éticas, as seis rações foram identificadas aleatoriamente por códigos: Ração A, Ração B, Ração C, Ração D, Ração E e Ração F (Tab. I).

As amostras foram transportados para o Laboratório de Entomologia Agrícola, Instituto de Biologia, Universidade Federal Rural do Rio de Janeiro, Seropédica, onde os insetos foram identificados e os péletes contados, separando os íntegros dos danificados. Diagnosticadas as espécies, foi utilizado o cálculo da porcentagem de infestação descrita por Nakano et al. (2002) em cada amostra de ração selecionada. Os espécimens iden- 


\section{Tabela I}

Comparação entre a composição nutricional dos seis diferentes tipos de rações industriais, fabricadas para cães e gatos, a venda em 2003 no comércio da cidade do Rio de Janeiro, Brasil.

Rações

Analisadas
Principais componentes e valores nutricionais indicados pelo fabricante

Ração A Milho integral moído, farinha de carne, farelo de soja, quirera de arroz, farinha de subprodutos de frango, farelo de trigo, farelo de glúten de milho, miúdos de aves e premix. U.12\% ; P.B. $27 \%$; E.E. $8,5 \%$; M.F. $4 \%$; M.M. $9 \%$.

Ração B Milho integral moído, quirera de arroz, farelo de soja, farinha de carne, farinha de subprodutos de frango, farelo de arroz, miúdos de aves hidrolisados, raízes de chicória, espinafre,cenoura, ervilha, premix vitamínico e premix mineralU.12\% ; P.B. $21 \%$; E.E. $9 \%$; M.F. 4,5\%; M.M. 8\%.

Ração C Farinha de vísceras, farinha de carne, levedura seca de cervejaria,milho integral, arroz integral, farelo de glúten de milho,cloreto de sódio (sal comum), gordura desidratada, espinafre desidratado, óleo de peixe, premix mineral e premix vitamínico.U.12\% ; P.B. $22 \%$; E.E. $6 \%$; M.F. $5 \%$; M.M. $8 \%$.

Ração D Farelo de glúten de milho, farelo de soja, levedura seca de cana de açúcar, milho integral moído, açúcar ,semente de linhaça, farinha de carne, arroz integral, batata desidratada, premix vitamínico e mineral.U. $12 \%$; P.B. $22 \%$; E.E. $6 \%$; M.F. $5 \%$; M.M. $8 \%$.

Ração E Milho integral moído, quirera de arroz, farelo de soja, farelo de trigo, farinha de peixe, farinha de carne e ossos e premix vitamínico.U.12\% ; P.B. $30 \%$; E.E. $8 \%$; M.F. $4,5 \%$; M.M. $8,5 \%$.

Ração F Carne bovina, carne de frango, extrato de carne, farinha, de carne, farinha de carne de frango, glúten de milho, trigo integral, arroz integral, milho integral moído, espinafre, óleo de girassol e levedura seca de cervejaria.U. $12 \%$; P.B. $19 \%$; E.E. $8 \%$; M.F. $4 \%$; M.M. $12 \%$.

U.= umidade da embalagem ; P.B.= proteína bruta ; E.E.= extrato etéreo; M.F.= matéria fibrosa ; M.M.= matéria mineral. 
tificados foram depositados na coleção entomológica do Laboratório de Entomologia Agrícola, Universidade Federal Rural do Rio de Janeiro.

\section{Resultados}

No material recolhido das seis marcas de rações industrializadas, registrou-se a incidência de 155 espécimens (Fig.1) das quais, 53 correspondem a (Figs. 2 e 3) Oryzaephilus surinamensis (Cucujidae) e 102 a espécie Necrobia rufipes (Cleridae).

A taxa percentual de infestação (Tab. II) entre as rações variou de 11,7 à $16 \%$. As rações $F, A$ e $B$ apresentaram taxa de infestação elevada, $(14,6-16 \%)$ e nas demais rações a taxa de infestação foi de 11,7 à 12,9\%.

A infestação de $O$. surinamensis e $N$. rufipes ocasionou a danificação em diversos péletes tornando-os fracos e quebradiços, em alguns casos o esfarelamento e a deterioração estavam visíveis, comprometendo assim, a qualidade dos produtos analisados. Trata-se, portanto, do primeiro registro destes besouros, infestando rações de animais domésticos no Brasil.

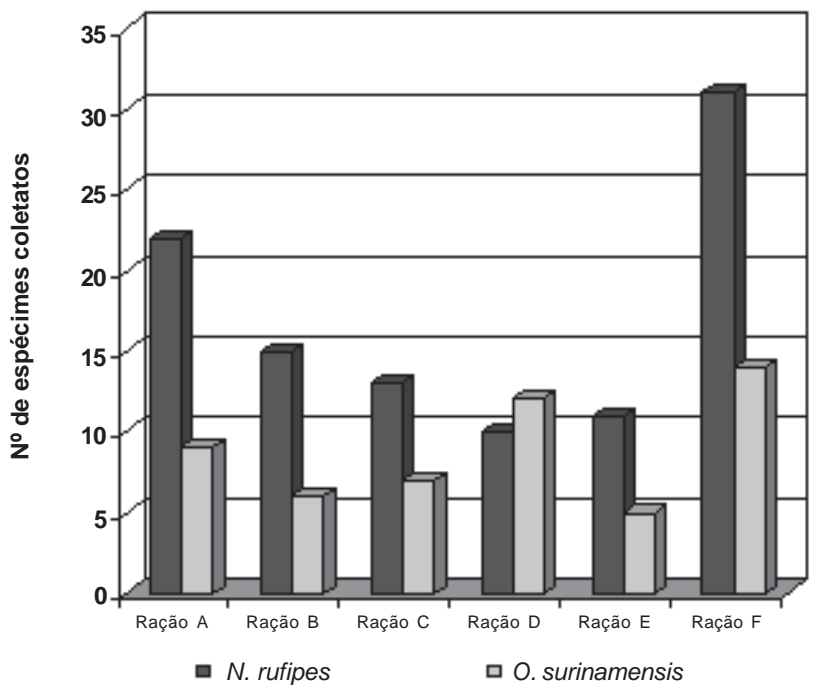

Figura 1: Insetos coletados nas diferentes rações para cães e gatos selecionadas no comércio da cidade do Rio de Janeiro em 2003. 


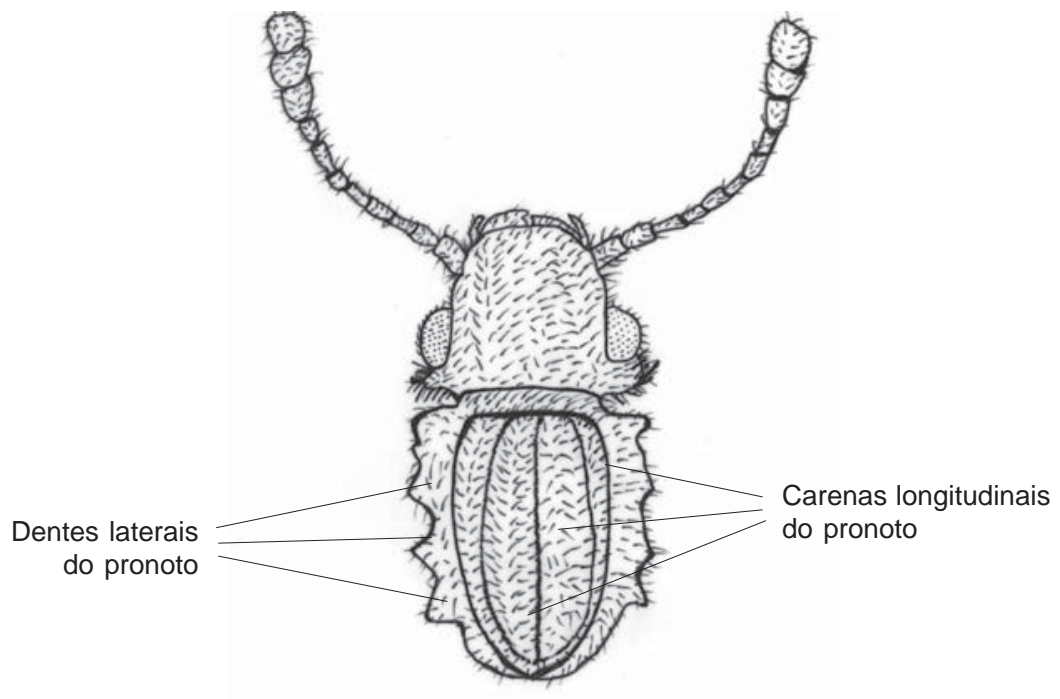

Figura 2: Oryzaephilus surinamensis (Coloptera:Cucujidae) - Vista dorsal da cabeça e tórax.

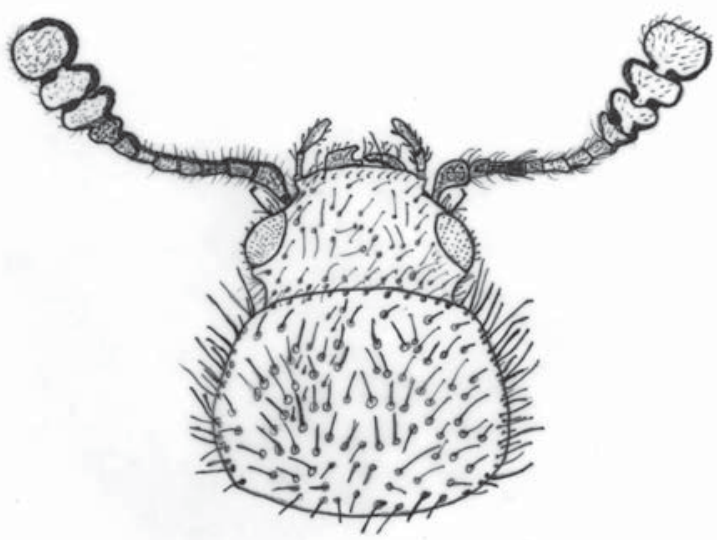

Figura 3: Necrobia rufipes (Coleoptera:Cleridae) - Vista dorsal da cabeça e tórax. 
Tabela II

Porcentagem entre o grau de infestação de Coleoptera (Insecta) nos péletes de seis diferentes tipos de ração, para cães e gatos, em 2003 no Rio de Janeiro, Brasil

\begin{tabular}{c|c|c|c|c|c}
\hline $\begin{array}{c}\text { Rações } \\
\text { selecionadas }\end{array}$ & $\begin{array}{c}\text { Peso por } \\
\text { amostra }\end{array}$ & $\begin{array}{c}\text { Total de } \\
\text { péletes }\end{array}$ & $\begin{array}{c}\text { Total de } \\
\text { péletes } \\
\text { íntegros }\end{array}$ & $\begin{array}{c}\text { Total de } \\
\text { péletes } \\
\text { danificados }\end{array}$ & $\begin{array}{c}\text { (\%) } \\
\text { Infestação }\end{array}$ \\
\hline Ração A & $1,5 \mathrm{~kg}$ & 9036 & 7635 & 1401 & 15,5 \\
Ração B & $1,5 \mathrm{~kg}$ & 3168 & 2706 & 462 & 14,6 \\
Ração C & $1,5 \mathrm{~kg}$ & 2934 & 2556 & 378 & 12,9 \\
Ração E & $1,5 \mathrm{~kg}$ & 5559 & 4846 & 713 & 12,8 \\
Ração F & $1,5 \mathrm{~kg}$ & 5811 & 4884 & 927 & 16,0 \\
\hline
\end{tabular}

\section{Discussão}

A diferença na taxa percentual da infestação nas rações selecionadas sugere há influência direta nas variedades de componentes nutricionais e dos níveis de proteína estabelecida em cada marca (Tab.I). A umidade de $12 \%$, padrão industrial nas embalagens das rações analisadas, favorece a proliferação destes insetos. Nakano et al. (2002) relatou que um percentual de umidade inferior a $10 \%$ prejudica o desenvolvimento de pragas e conseqüentemente os danos aos produtos armazenados diminuem consideravelmente.

A infestação pode ocasionar perdas econômicas significativas às indústrias tal como foi assinalado por Osuji (1977). Danos gerados nos péletes podem reduzir os componentes nutricionais, principalmente as proteínas, já que estas são substratos importantes para o desenvolvimento biológico destes coleópteros, sendo encontradas em abundância nas rações de animais domésticos (Ashman,1963; Sengupta et al., 1984).

A omissão no controle sanitário do processamento das rações reforça a preocupação dos cuidados que devem ser realizados quanto à produção de alimentos industrializados. No entanto, a origem da infestação permanece em questão, visto que pode ser decorrente da matéria prima fornecida as indústrias ou da área destinada ao processo de fabricação. 


\section{Agradecimentos}

Ao professor adjunto do Laboratório de Entomologia Agrícola da UFRuralRJ, Francisco Racca Filho pelo auxílio na identificação da espécie Necrobia rufipes.

\section{Referências Bibliográficas}

Ashman, F., 1963. Factors affecting the abundance of the copra beetle, Necrobia rufipes (Deg.) (Coleoptera, Cleridae). Bull. Entomol. Res. 53:671-680.

Baggio, D.; Figueiredo, S.M.; Flechtmann, C.H.W.; Zambon, G.Q. \& Miranda, S.H.G., 1987. Avaliacão de presenca de acaros em cereais armazenados na grande São Paulo. An. Esc. Sup. Agric. 44:617-626

Buchelos C.T. \& Athanassiou C.G., 1999. Unbaited probe traps and grain trier: a comparison of the two methods for sampling Coleoptera in stored barley. J. Stored Prod. Res. 35:397-404.

Carvalho, L.M.L; Thyssen, P.J; Linhares, A.X. \& Palhares, F.A.B., 2000. A checklist of arthropods associated with pig carrion and human corpses in Southeastern Brazil. Mem. Inst. Oswaldo Cruz. 95:135-138.

Curtis,C.E. \& Clark,J.D., 1974. Comparative biologies of Oryzaephilus surinamensis and mecartor (Coleoptera:Cucujidae) on dried fruits and nuts. U.S. Dep. Agric. Tech. Bull. 1488:1-42.

Fields, P., 1992. The control of stored-products insects and mites with extreme temperatures. J. Stored Prod. Res. 28:89-118.

Howe,R.W., 1956. Thexbiology of the two common storage species of Oryzaephilus (Coleoptera:Cucujidae). Ann. Appl. Biol. 44:341-342

Lambkin, T.A. \& Khatoon N., 1990. Culture methods for Necrobia rufipes (De Geer) and Dermestes maculatus De Geer (Coleoptera:Cleridae and Dermestidae). J. Stored Prod. Res. 26:59-60. 
Nakano, O.; Neto, S.S.; Carvalho, R.P. L.; Baptista, G.C. de.; Filho, E.B.; Parra, J.R.P.; Zucchi, R.A.; Alves, S.B.; Vedramim, J.D.; Marchini, L.C.; Lopes, J.R.S. \& Omoto, C., 2002. Entomologia/Agrícola, Fealq, São/ Paulo. 920 p.

Nwana, I.E.A., 1993. Survey of storage coleoptera with attack dried cocoyam chips in Nigeria. J. Stored Prod. Res. 29:95-98.

Odeyemi, O.O., 1997. Interspecific competition between the beetles Dermestes maculatus DeGeer and Necrobia rufipes DeGeer in dried fish. Insect Sci. Applic. 17:213-220.

Osuji, F.N.C., 1977. The development of Necrobia rufipes in dried fish and certain other commodities. Niger. J. Sci. 15:21-32.

Pratissoli, D., 1997. Ocorrência de Necrobia rufipes atacando castanha de caju proviniente da Índia. An. Soc. Entomol. Bras. 26:573-574.

Sengupta, T.; Mukhopadhyay, P. \& Sengupta, R., 1984. Major beetle pest of stored food products in India. Records Zool. Surv. India Occas. 62:65-66

Shalaby, O.A.; de Carvalho, L.M.L..\& Goff, M.J., 2000. Comparison of patterns of decomposition in hanging carcass and a carcass in contact with soil in a xerophytic habitat on the island of Oahu, Hawaii. J. Forensic. Sci. 45:1267-1273.

Simmons, P.\& Ellington, G.W., 1925. The ham beetle, Necrobia rufipes DeGeer. J. Agric. Res. 30:845-863.

Veer, V.; Negi, B.K. \& Rao, K.M., 1996. Dermestid beetles and some other insects pests associated with stored silkworm cocoons in India, including a world list of dermestid species found attacking this commodity. J. Stored Prod. Res. 32:69-89. 